\title{
The effects of a single-dose subacromial injection of a nonsteroidal anti-inflammatory drug in geriatric patients with subacromial impingement syndrome: a randomized double- blind study
}

\author{
Young Bae Kim, Woo-Seung Lee, Jun-Sung Won \\ Department of Orthopedic Surgery, Veterans Health Service Medical Center, Seoul, Korea
}

\begin{abstract}
Background: As nonsteroidal anti-inflammatory drugs (NSAIDs) and steroids have similar effects, steroids can be avoided to reduce adverse effects. This study aimed to compare the differences in symptom improvement after subacromial injection of steroids or NSAIDs. Methods: Sixty patients with rotator cuff syndrome for at least 3 months were enrolled and divided into steroid and NSAID groups. The steroid group received a mixture of $1 \mathrm{~mL}$ of triamcinolone acetonide $(40 \mathrm{mg} / \mathrm{mL})$ and $1 \mathrm{~mL}$ of lidocaine hydrochloride $2 \%$, while the NSAID group received a mixture of $1 \mathrm{~mL}$ of Ketorolac Tromethamine $(30 \mathrm{mg} / \mathrm{mL})$ and $1 \mathrm{~mL}$ of lidocaine hydrochloride $2 \%$. The patients were assessed before and at 3, 6, and 12 weeks after the procedure. Shoulder scores from visual analog scale (VAS), American Shoulder and Elbow Surgeons (ASES), and University of California Los Angeles (UCLA) were used for evaluation.

Results: Both groups showed improvements in the clinical outcomes. Overall VAS, ASES, and UCLA scores improved from 6.9, 32.7, and 16.0 before the procedure to $2.0,1.2$, and $1.1 ; 81.5,87.6$, and 88.5 ; and $29.7,31.8$, and 32.0 at weeks 3,6 , and 12 weeks after the procedure, respectively. Twenty-six patients (86.7\%) in the steroid group and $28(93.3 \%)$ in the NSAID group reported satisfactory treatment outcomes. There were no significant differences in the outcomes between the two groups $(\mathrm{p}=0.671)$.

Conclusions: Subacromial injection of NSAIDs for rotator cuff tendinitis with shoulder pain had equivalent outcomes with those of steroid injection at the 12-week follow-up.
\end{abstract}

Keywords: Anti-inflammatory agents, non-steroidal; Impingement syndrome; Ketorolac; Rotator cuff injuries; Triamcinolone

\section{INTRODUCTION}

The most common cause of shoulder pain is impingement syndrome in the subacromial space, which consists of the rotator cuff, bursa, and coracoacromial ligament [1]. Impingement between the humeral head and coracoacromial arch is associated with osteophytes within the acromion, weakening of the rotator cuff, and mismatch of shoulder movements [2]. Various treatment modalities, including medication, physical therapy, intra-articular injection, and operative decompression, are used to treat impingement syndrome [3].

Intra-articular injections are easily performed in outpatients.

Received: January 25, $2021 \quad$ Revised: February 15, $2021 \quad$ Accepted: February 16, 2021

Correspondence to: Jun-Sung Won

Department of Orthopedic Surgery, Veterans Health Service Medical Center, 53 Jinhwangdo-ro 61-gil, Gangdong-gu, Seoul 05368, Korea

Tel: +82-2-2225-1352, Fax: +82-2-2225-1910, E-mail: 99korean@naver.com, ORCID: https://orcid.org/0000-0002-5454-4877

Financial support: None.

Conflict of interest: None.

Copyright@ 2021 Korean Shoulder and Elbow Society. All Rights Reserved.

This is an Open Access article distributed under the terms of the Creative Commons Attribution Non-Commercial License (http://creativecommons.org/licenses/by-nc/4.0/) which permits unrestricted non-commercial use, distribution, and reproduction in any medium, provided the original work is properly cited. 
Corticosteroids have been widely used in intra-articular injections for treatment of impingement syndrome because of their strong anti-inflammatory effects [4-8]. However, repeated use of steroids is not advisable due to resulting possible tears and weakening of the ligament or tendon in the shoulder, delayed healing, decrease in immunity, increased incidence of bacterial infection, and fluctuating blood glucose level [9-12].

Nonsteroidal anti-inflammatory drugs (NSAIDs) and platelet-rich plasma have been proposed as injectable options [13-15]. NSAIDs have been widely used, and their effectiveness in reducing pain and inflammation, in addition to their stability when administered orally or as intramuscular injections, has been demonstrated. Several authors have reported that an intra-articular ketorolac injection is effective and safe for articular cartilage, ligament, and joint function [14,16]. However, the exact method of injection and the effective dose are unknown.

The aim of this prospective, double-blinded study was to evaluate the short-term effect of subacromial injection of NSAIDs compared with the outcomes of steroid injections. If NSAIDs have effects equivalent to those of steroids, the adverse effects associated with steroids could be avoided with the use of NSAIDs. The exact method of injection for geriatric patients was established, and three established score systems were adopted for evaluation.

\section{METHODS}

\section{Demographic Data}

The data were obtained from patients with subacromial impingement syndrome who were treated at our medical center from March 2016 to December 2017, after approval from the Institutional Review Board (IRB No. 2016-03-019). Informed consent was received before data collection.

A randomized, prospective, case-control study was conducted. The sizes of the experimental group and control group were determined based on a previous study considering a change in visual analog scale (VAS) score for pain greater than 20\% [17,18]. A power analysis was used to calculate the required sample size. Assuming an approximate normal distribution for the primary outcome measure and a standard deviation of 15 points, to detect a 15-point difference in the Constant-Murley Shoulder score between treatment groups at a $5 \%$ level of significance with $80 \%$ power. Allowing for some losses to follow up (5\%), a minimum sample size of 30 patients was needed for each arm of the trial.

Patients were enrolled if all the following inclusion criteria were satisfied: shoulder pain for more than 3 months, shoulder pain while lifting the arm between $70^{\circ}$ and $120^{\circ}$, and positive re- sults either for Neer's test or Hawkin's test. The exclusion criteria were as follows: osteoarthritis, calcific tendonitis, visible fracture on shoulder radiographs, passive anterior elevation less than $90^{\circ}$ or external rotation less than $20^{\circ}$, history of an injection in the shoulder within the last 6 months, or rotator cuff tear on magnetic resonance imaging (MRI). Patients with uncontrolled diabetes, past history of gastritis or treatment for gastric bleeding, contraindications to steroids or NSAIDs, or secondary benefits from accidents or insurance also were excluded.

The participants were divided into two groups (group $\mathrm{N}$ received an NSAID injection and group S received a steroid injection) according to the following randomization procedure. Thirty cards of groups A and B were placed and sealed in a box, and the patient selected a card without the examiner's knowledge. There were no differences in age, sex, duration of illness, diabetes, VAS score, American Shoulder and Elbow Surgeons (ASES) shoulder score, and University of California Los Angeles (UCLA) shoulder score between the two groups (Table 1).

\section{Treatment Method}

A subacromial injection as indicated on the chosen card was administered with an opaque syringe in an injection room. The physicians who administered the injection were not involved in the rest of the study. One milliliter of ketorolac (ketorolac tromethamine $30 \mathrm{mg} / \mathrm{mL}$ ) or $1 \mathrm{~mL}$ of steroid (triamcinolone $40 \mathrm{mg} /$ $\mathrm{mL}$ ) and $1 \mathrm{~mL}$ of lidocaine (2\% lidocaine hydrochloride inj.) in an opaque syringe was administered depending on group.

With the patient sitting comfortably, the upper arm was placed in $10^{\circ}$ abduction and $10^{\circ}$ lifting. An assistant held the arm in position while the injection was administered. After sterilization with alcohol and povidone, a $5 \mathrm{~mL}$ syringe with a $21-\mathrm{G}$ needle was filled with the drug, and the needle was advanced to the rear of the acromioclavicular joint, $2-3 \mathrm{~cm}$ from the lateral center of

Table 1. Patient demographics by injection group

\begin{tabular}{lccc}
\hline Variable & Group N & Group S & p-value \\
\hline Number & 30 & 30 & \\
Age (yr) & $66.6 \pm 6.0$ & $68.8 \pm 6.0$ & 0.151 \\
Sex (male:female) & $19: 11$ & $22: 8$ & 0.405 \\
Symptom duration (mo) & $8.8 \pm 7.2$ & $7.4 \pm 5.5$ & 0.388 \\
DM & 5 & 4 & 0.718 \\
VAS pain score & $6.8 \pm 0.4$ & $6.9 \pm 0.4$ & 0.356 \\
ASES score & $33.1 \pm 8.9$ & $31.9 \pm 8.7$ & 0.595 \\
UCLA score & $40.0 \pm 9.3$ & $38.5 \pm 9.0$ & 0.706 \\
\hline
\end{tabular}

Values are presented as mean \pm standard deviation.

Group N: ketorolac injection group, Group S: steroid injection group, DM: diabetes mellitus, VAS: visual analog scale, ASES: American Shoulder and Elbow Surgeons, UCLA: The University of California, Los Angeles. 
the acromion, in the inferior direction. After aspiration to ensure that the needle was not inside a vessel, the injection was administered. If resistance was felt during the injection, the procedure was immediately stopped, the needle was changed, and the injection re-administered with absence of resistance. During the study, all patients were prescribed acetaminophen and were trained to perform stretching exercises at home. The stretching exercises comprised lift, external rotation, internal rotation, and adduction exercises to an extent such that resistance was felt, and the position was maintained for 10 seconds. This was repeated 20 times for each exercise, four times a day before breakfast, lunch, dinner, and going to bed.

\section{Assessment or Outcomes}

Clinical evaluation was performed before treatment and at 3, 6, and 12 weeks after treatment. VAS pain, ASES, and UCLA scores were assessed [19]. Patient satisfaction was assessed at the final follow-up using a numeric scale, with one point indicating the highest level of dissatisfaction and 10 points indicating the highest level of satisfaction. A score of 8 or higher was considered satisfactory. Range of motion is included in the shoulder score. Also, function was considered more important than height of arm lift. Therefore, the study focused on shoulder score.

For statistical analysis, t-test, chi-square test, and Fisher's exact test were used with IBM SPSS ver. 22.0 (IBM Corp., Armonk, $\mathrm{NY}$, USA), and statistical significance was set at $\mathrm{p}$-value $\leq 0.05$.

\section{RESULTS}

There were no differences in age, sex, duration of pain, VAS score, ASES score, and UCLA score between the two groups before treatment. VAS, ASES, and UCLA scores improved in both groups from week three to week 12 after treatment $(p<0.000)$. In all patients, VAS score improved from 6.9 before treatment to 2.0, 1.2 , and 1.1 at 3,6 , and 12 weeks after treatment, respectively. ASES score improved from 32.7 before treatment to 81.5, 87.6, and 88.5 at 3, 6, and 12 weeks after treatment, respectively. UCLA score improved from 16.0 before treatment to $29.7,31.8$, and 32.0 at 3, 6, and 12 weeks after treatment, respectively.

In the NSAID group, pain VAS score improved from $6.8 \pm 0.4$ before treatment to $1.8 \pm 1.7,1.1 \pm 1.8$, and $0.9 \pm 1.8$ at 3,6 , and 12 weeks after treatment, respectively, and in the steroid group, VAS score improved from $6.9 \pm 0.4$ before the treatment to $2.2 \pm 1.7$, $1.3 \pm 1.7$, and $1.3 \pm 2.1$ at 3,6 , and 12 weeks after treatment. There was no statistical difference between the two groups at each follow-up visit ( $\mathrm{p}=0.356, \mathrm{p}=0.452, \mathrm{p}=0.715, \mathrm{p}=0.469)$ (Table 2). ASES score in the NSAID group improved from $33.1 \pm 8.9$ before treatment to $83.1 \pm 15.0,88.1 \pm 16.2$, and $90.3 \pm 15.9$ at 3,6 , and 12 weeks after treatment respectively, and in the steroid group, it improved from $31.9 \pm 8.7$ before treatment to $80.1 \pm 15.3$, $87.4 \pm 16.5$, and $87.0 \pm 20.1$ at 3,6 , and 12 weeks after treatment, respectively. There was no statistical difference between the two groups at each follow-up visit $(\mathrm{p}=0.595, \mathrm{p}=0.447, \mathrm{p}=0.868$, $\mathrm{p}=0.475$ ) (Table 2). UCLA score in the NSAID group improved from $16.0 \pm 1.4$ before treatment to $30.8 \pm 5.3,32.4 \pm 5.3$, and $33.2 \pm 5.0$ at 3,6 , and 12 weeks after treatment, respectively, and in the steroid group, it improved from $15.9 \pm 1.4$ to $28.7 \pm 4.5$, $31.2 \pm 4.9$, and $31.0 \pm 5.9$. There was no statistical difference between the two groups at each follow-up visit $(p=0.706, p=0.111$, $\mathrm{p}=0.367, \mathrm{p}=0.144$ ) (Table 2). The average patient satisfaction score after injection in the steroid group was $8.6 \pm 2.1$, and that in the NSAID group was $8.9 \pm 1.9$; there was no statistical difference between the two groups $(\mathrm{p}=0.565)$. Satisfactory results were obtained in 26 cases $(86.7 \%)$ in the steroid group and 28 cases (93.3\%) in the NSAID group. There was no statistical difference between the two groups $(\mathrm{p}=0.671)$.

There were no cases with shoulder infection after subacromial injection; however, in one case, diabetes remained uncontrolled for 1 month after corticosteroid injection, and two patients complained of facial flushing. The participant with uncontrolled diabetes was treated at the hospital's endocrine department. The diabetes was well controlled until before treatment, and the glycosylated hemoglobin (HbA1c) level was 6.2\%. After 1 month, the diabetes was under control without a change in medication. Both

Table 2. Clinical scores by injection method

\begin{tabular}{llll}
\hline Variable & Group N & Group S & p-value \\
\hline VAS pain score & & & \\
Pre-injection & $6.8 \pm 0.4$ & $6.9 \pm 0.4$ & 0.356 \\
3 wk & $1.8 \pm 1.7$ & $2.2 \pm 1.7$ & 0.452 \\
6 wk & $1.1 \pm 1.8$ & $1.3 \pm 1.7$ & 0.715 \\
12 wk & $0.9 \pm 1.8$ & $1.3 \pm 2.1$ & 0.469 \\
ASES score & & & \\
Pre-injection & $33.1 \pm 8.9$ & $31.9 \pm 8.7$ & 0.595 \\
3 wk & $83.1 \pm 15.0$ & $80.1 \pm 15.3$ & 0.447 \\
6 wk & $88.1 \pm 16.2$ & $87.4 \pm 16.5$ & 0.868 \\
12 wk & $90.3 \pm 15.9$ & $87.0 \pm 20.1$ & 0.475 \\
UCLA score & & & \\
Pre-injection & $16.0 \pm 1.4$ & $15.9 \pm 1.4$ & 0.706 \\
3 wk & $30.8 \pm 5.3$ & $28.7 \pm 4.5$ & 0.111 \\
6 wk & $32.4 \pm 5.3$ & $31.2 \pm 4.9$ & 0.367 \\
12 wk & $33.2 \pm 5.0$ & $31.0 \pm 5.9$ & 0.144 \\
\hline
\end{tabular}

Values are presented as mean \pm standard deviation.

Group N: ketorolac injection group, Group S: steroid injection group, VAS: visual analog scale, ASES: American Shoulder and Elbow Surgeons, UCLA: The University of California, Los Angeles. 
cases of facial flushing were women, and symptoms persisted for one week and improved without any treatment.

\section{DISCUSSION}

This randomized, controlled, comparative study showed that ketorolac $30 \mathrm{mg} / \mathrm{mL}$ was not inferior to steroids as a subacromial injection for pain relief in patients with subacromial impingement syndrome. VAS pain, ASES, and UCLA scores were not different between the two groups, and the adverse effects of steroids were avoided using ketorolac.

The advantage of ketorolac is that its half-life is 150 minutes, which is longer than 88 minutes in triamcinolone, and is expected to be more effective for anti-inflammatory action [18]. Systemic adverse effects and stability of NSAIDs such as ketorolac administered as intramuscular and intravascular injections are well-known. The risks of intra-articular injection are not widely known; however, in animal experiments on rats and rabbits, intra-articular injection of NSAIDs did not cause cartilaginous changes, indicating its relative safety $[16,20]$.

The most common adverse effect of steroids is flushing of the face, reported in $40 \%$ of patients [21]. In this study, two of $30 \mathrm{pa-}$ tients experienced flushing, both females, and the symptom resolved after 1 week without additional treatment. However, as the probability of recurrence of flushing during repeat injection is reported to be up to $100 \%$, it is important to monitor patient discomfort closely and to prevent recurrence of the same adverse effects [21].

Topical corticosteroid injections can lead to elevated blood glucose level in diabetic patients [11]. In this study, blood glucose remained uncontrolled in one case but improved after 1 month without a change in medication. Additional tests showed no significant increase in glycated hemoglobin level; nevertheless, special attention and care are needed.

Three or more repeated corticosteroid injections increase the rate of suture failure by decreasing the tendon suture displacement strength during rotator cuff repair surgery [22]. However, there is no evidence of absence of reduction in suture displacement strength with NSAIDs. Nevertheless, since there is no report of muscular atrophy after intramuscular injection of NSAIDs, they are thought to be safer than steroids. According to Almekinder et al. [23], NSAIDs have a positive effect on the maturation and reforming stages of tendon healing.

Limitations of using NSAIDs include the probability of decreased renal function and gastritis. However, in cases of subacromial impingement, they are not used continuously, unlike when taken as oral medications for other conditions. Moreover, a topical injection is thought to have a less severe effect on renal function compared to oral administration.

Another limitation is the possibility that adhesive capsulitis cases were included in the study. Patients with less than $90^{\circ}$ of anterior elevation and less than $20^{\circ}$ of external rotation were excluded, but not all patients with stiff shoulder were excluded. However, it is not expected that there will be a significant impact on the results.

In this study, the follow-up period was short (3 months); hence, the long-term effectiveness and adverse effects of treatment were not assessed. Furthermore, we were unable to investigate the status of the rotator cuff using MRI or arthroscopy in many patients. However, conservative therapy can be administered as an initial treatment for a rotator cuff tear, which does not cause any harm, such as a delay in treatment, and is thought to be clinically useful. Another limitation is that ultrasonography was not used to guide subacromial injections, which might affect the accuracy of the location of injections. Finally, the patients were instructed to perform exercises at home. These are important for conservative treatment but could not be verified in all patients. Since the treatment result was satisfactory, high compliance was assumed.

Another limitation is that the effects of acetaminophen cannot be controlled. However, in patients with subacromial impingement syndrome, acetaminophen is commonly administered as a subacromial injection to reduce pain and is not a problem for clinical use. Despite these limitations, the strengths of the study include no loss of participants due to dropout and absence of associated errors. In treatment of subacromial impingement syndrome, subacromial injection of Ketorolac $30 \mathrm{mg}$ was equivalent to corticosteroid injection in terms of effectiveness. In patients with diabetes or those who have concerns about the adverse effects of steroids, subacromial injection of Ketorolac can be considered a suitable alternative.

\section{ORCID}

Youngbea B Kim

https://orcid.org/0000-0003-3966-0949

Jun-Sung Won

https://orcid.org/0000-0002-5454-4877

\section{REFERENCES}

1. Michener LA, McClure PW, Karduna AR. Anatomical and biomechanical mechanisms of subacromial impingement syndrome. Clin Biomech (Bristol, Avon) 2003;18:369-79.

2. Harrison AK, Flatow EL. Subacromial impingement syndrome. J Am Acad Orthop Surg 2011;19:701-8. 
3. Koester MC, George MS, Kuhn JE. Shoulder impingement syndrome. Am J Med 2005;118:452-5.

4. Cakmak A. Conservative treatment of subacromial impingement syndrome. Acta Orthop Traumatol Turc 2003;37 Suppl 1:112-8.

5. Cole BJ, Schumacher HR Jr. Injectable corticosteroids in modern practice. J Am Acad Orthop Surg 2005;13:37-46.

6. Hiemstra LA, Macdonald PB, Froese W. Subacromial infection following corticosteroid injection. J Shoulder Elbow Surg 2003; 12:91-3.

7. Holt TA, Mant D, Carr A, et al. Corticosteroid injection for shoulder pain: single-blind randomized pilot trial in primary care. Trials 2013;14:425.

8. van der Windt DA, Koes BW, de Jong BA, Bouter LM. Shoulder disorders in general practice: incidence, patient characteristics, and management. Ann Rheum Dis 1995;54:959-64.

9. Lee HJ, Kim YS, Ok JH, Lee YK, Ha MY. Effect of a single subacromial prednisolone injection in acute rotator cuff tears in a rat model. Knee Surg Sports Traumatol Arthrosc 2015;23:55561.

10. Ramírez J, Pomés I, Cabrera S, Pomés J, Sanmartí R, Cañete JD. Incidence of full-thickness rotator cuff tear after subacromial corticosteroid injection: a 12-week prospective study. Mod Rheumatol 2014;24:667-70,

11. Stepan JG, London DA, Boyer MI, Calfee RP. Blood glucose levels in diabetic patients following corticosteroid injections into the hand and wrist. J Hand Surg Am 2014;39:706-12.

12. Tillander B, Franzén LE, Karlsson MH, Norlin R. Effect of steroid injections on the rotator cuff: an experimental study in rats. J Shoulder Elbow Surg 1999;8:271-4.

13. Shams A, El-Sayed M, Gamal O, Ewes W. Subacromial injection of autologous platelet-rich plasma versus corticosteroid for the treatment of symptomatic partial rotator cuff tears. Eur J Orthop Surg Traumatol 2016;26:837-42.

14. Goyal T, Paul S, Sethy SS, Choudhury AK. Outcomes of ketorolac versus depomedrol infiltrations for subacromial impingement syndrome: a randomized controlled trial. Musculoskelet
Surg 2020 May 22 [Epub]. https://doi.org/10.1007/s12306-02000667-7.

15. Elzaher EH, Kotb AM, Hassaan EH. Ultrasound guided injection of platelet enriched plasma versus corticosteroids in patients suffering rotator cuff tendinopathy. QJM 2020;113:hcaa059010.

16. Dogan N, Erdem AF, Gundogdu C, Kursad H, Kizilkaya M. The effects of ketorolac and morphine on articular cartilage and synovium in the rabbit knee joint. Can J Physiol Pharmacol 2004; 82:502-5.

17. Karthikeyan S, Kwong HT, Upadhyay PK, Parsons N, Drew SJ, Griffin D. A double-blind randomised controlled study comparing subacromial injection of tenoxicam or methylprednisolone in patients with subacromial impingement. J Bone Joint Surg Br 2010;92:77-82.

18. Min KS, St Pierre P, Ryan PM, Marchant BG, Wilson CJ, Arrington ED. A double-blind randomized controlled trial comparing the effects of subacromial injection with corticosteroid versus NSAID in patients with shoulder impingement syndrome. J Shoulder Elbow Surg 2013;22:595-601.

19. Oh JH, Kim SH, Lee HK, Jo KH, Bin SW, Gong HS. Moderate preoperative shoulder stiffness does not alter the clinical outcome of rotator cuff repair with arthroscopic release and manipulation. Arthroscopy 2008;24:983-91.

20. Jean YH, Wen ZH, Chang YC, et al. Intra-articular injection of the cyclooxygenase-2 inhibitor parecoxib attenuates osteoarthritis progression in anterior cruciate ligament-transected knee in rats: role of excitatory amino acids. Osteoarthritis Cartilage 2007;15:638-45.

21. Pattrick M, Doherty M. Facial flushing after intra-articular injection of steroid. Br Med J (Clin Res Ed) 1987;295:1380.

22. Blair B, Rokito AS, Cuomo F, Jarolem K, Zuckerman JD. Efficacy of injections of corticosteroids for subacromial impingement syndrome. J Bone Joint Surg Am 1996;78:1685-9.

23. Almekinders LC, Banes AJ, Ballenger CA. Effects of repetitive motion on human fibroblasts. Med Sci Sports Exerc 1993;25: 603-7. 